\title{
The optimized Rayleigh-Ritz scheme for determining the quantum-mechanical spectrum
}

\author{
Przemysław Kościk and Anna Okopińska \\ Institute of Physics, Świętokrzyska Academy, Świętokrzyska 15, 25-406 Kielce, Poland
}

Received 3 April 2007, in final form 4 July 2007

Published 14 August 2007

Online at stacks.iop.org/JPhysA/40/10851

\begin{abstract}
The convergence of the Rayleigh-Ritz method with nonlinear parameters optimized through minimization of the trace of the truncated matrix is demonstrated by a comparison with analytically known eigenstates of various quasi-solvable systems. We show that the basis of the harmonic oscillator eigenfunctions with optimized frequency $\Omega$ enables determination of boundstate energies of one-dimensional oscillators to an arbitrary accuracy, even in the case of highly anharmonic multi-well potentials. The same is true in the spherically symmetric case of $V(r)=\frac{\omega^{2} r^{2}}{2}+\lambda r^{k}$, if $k>0$. For spiked oscillators with $k<-1$, the basis of the pseudoharmonic oscillator eigenfunctions with two parameters $\Omega$ and $\gamma$ is more suitable, and optimization of the latter appears crucial for a precise determination of the spectrum.
\end{abstract}

PACS numbers: 03.65.Ge, 31.15.Pf, 02.70.Hm

\section{Introduction}

Since the early days of quantum mechanics, the techniques based on the variational principle have been successfully used for determination of ground-state characteristics of various physical systems. In its simplest form, the variational approach utilizes a single trial function that depends upon certain parameters, the values of which are fixed so as to minimize the expectation value of the Hamiltonian. This approach enables a very accurate determination of the ground-state energy, if the functional form of the trial function is appropriately chosen, but its extension to higher bound states appears impractical because of difficulties in assuring orthogonality of the trial functions. A more suitable approach is offered by the linear RayleighRitz (RR) method with a trial function represented as a finite linear combination

$$
\phi(\vec{r})=\sum_{n=0}^{N-1} c_{n} \psi_{n}(\vec{r}),
$$


where the functions $\psi_{n}(\vec{r})$ are taken from some orthonormal basis in the function space. Treating the coefficients $c_{n}$ as variational parameters, one obtains a set of linear equations

$$
\sum_{n=0}^{N-1}\left(H_{m n}-\varepsilon \delta_{m n}\right) c_{n}=0, \quad m=0,1, \ldots, N-1,
$$

where $H_{m n}=\langle m|\widehat{H}| n\rangle$ denotes the matrix element of the Hamiltonian between the states of the basis, $|n\rangle=\psi_{n}$. The solution of the above equations yields $N$ th order approximations to wavefunctions $\phi_{i}^{(N)}(\vec{r})$, and the corresponding energies $\varepsilon_{i}^{(N)}$, for $i=0, \ldots, N-1$ states. The accuracy of the RR method can be systematically improved by increasing the number $N$ of basis functions, obtaining successive approximations to the larger and larger number of states. In this way a desired part of the spectrum may be determined with the approximate eigenvalues monotonically converging to the exact bound-state energies [1] at a rate strongly dependent on the choice of the basis. The method is also known under the name of exact diagonalization although the results become exact only in the limit as $N$ tends to infinity. Long time ago, Hylleraas observed [2] that the effectiveness of the RR method may be further improved by introducing a scale parameter into the functions of the basis. Since then, various sets of functions depending on various nonlinear parameters have been used in determining spectra of atoms and molecules. The orthonormal sets made up of the eigenfunctions of a solvable Hamiltonian which depends on certain free parameters are especially convenient. In chemical applications the values of nonlinear parameters are fixed so as to optimize the convergence of the RR estimate to the ground-state energy; in the early works, the best values were found by trial and error [3] at present, they are fixed in computationally demanding procedures of iterative optimization $[4,5]$.

Optimization of the RR scheme may performed on the basis of the principle of minimal sensitivity (PMS), which has been successfully used to improve perturbative calculation [6-8]. PMS requires the values of unphysical parameters introduced into calculation to be chosen so as to make approximations to physical quantities as less sensitive to the variation of these parameters as possible. This suggests to improve the RR method by fixing the values of nonlinear parameters so as to minimize the $N$ th order approximation to the desired level energy $E_{n}^{N}$. Such a strategy requires however diagonalization of the RR matrix to be performed in an algebraic way, which is feasible but only in low orders [5], or the application of extensive procedures of iterative minimization. Moreover, the scheme is not very economical, since the whole procedure must be repeated for each considered state. The optimized RR scheme, proposed by one of us [9], adopts a different strategy that is also based on the PMS but insists on fixing the values of nonlinear parameters before diagonalization of the truncated matrix. Since the only physical quantity that can be determined before diagonalization of the $N$ th order RR matrix is its trace

$$
\operatorname{Tr}_{N} H=\sum_{n=0}^{N-1}\langle n|\widehat{H}| n\rangle,
$$

which represents the sum of $N$ bound-state energies, we require the values of nonlinear parameters to be chosen so as to render $\operatorname{Tr}_{N} H$ stationary. The advantage of the scheme is that the $N$ th order approximations to many eigenstates are determined in one run and the obtained approximations to wavefunctions are orthogonal. It has been shown [9] that a good accuracy is acquired for the quartic anharmonic oscillator, using a basis of the harmonic oscillator (HO) eigenfunctions with an optimized frequency. In this work we show that also in the case of other interaction potentials, the stationarity of the $\operatorname{Tr}_{N} H$ condition optimizes the choice of nonlinear parameters. We note that implementation of the method with a modern 
software environment allows arbitrary precision calculations, which is especially important for determining the tiny energy splitting in the case of multi-well potentials with nearly degenerate levels. The computational cost of the optimized RR scheme is not high, as the 50-digits accuracy of lowest bound-state energies is easily achieved with RR matrices of order $N<100$, even in the most difficult cases of multi-well oscillators. Through the example of a sextic oscillator with analytically known eigenstates we show that the performance of the method for wavefunctions is also good, as various moments of the position operator appear well convergent. Later, we discuss the case of spherically symmetric potentials, where the two-parameter set of pseudo-harmonic oscillator (PHO) eigenfunctions constitutes a more appropriate basis for the optimized RR method. We gauge the convergence of the method for various anharmonic potentials $\lambda r^{k}$, of both positive and negative power $k$, comparing the results with the exact solutions of different quasi-solvable examples (the sextic oscillator, the harmonium system and the spiked oscillator). In all the cases studied, a good convergence is automatically ensured by using the trace condition for fixing the nonlinear parameters.

The plan of our work is as follows. In section 18, we study the optimized RR method for one-dimensional anharmonic oscillators using the basis of the $\mathrm{HO}$ eigenfunctions. In section 3, the basis of the PHO eigenfunctions is introduced and the performance of the method for spherically symmetric potentials is discussed. Section 4 is devoted to conclusion.

\section{One-dimensional case}

\subsection{Harmonic oscillator basis}

The success of the RR method depends on the appropriate choice of the basis in the function space. A basis constructed from the HO eigenfunctions

$$
\psi_{n}^{\Omega}(x)=\left(\frac{\Omega}{\sqrt{\pi} 2^{n} n !}\right)^{\frac{1}{2}} H_{n}(\Omega x) \mathrm{e}^{-\frac{\Omega^{2} x^{2}}{2}}
$$

with an arbitrary frequency $\Omega$ playing a role of a nonlinear parameter has proved convenient for solving one-dimensional problems with a purely discrete spectrum [10-19]. We use the above basis for determining spectrum of various anharmonic oscillators (AO).

\subsection{Quartic oscillator}

The most popular example is the quartic AO with the Hamiltonian operator given by

$$
\widehat{H}=-\frac{1}{2} \frac{\mathrm{d}^{2}}{\mathrm{~d} x^{2}}+\frac{\omega^{2} x^{2}}{2}+\lambda x^{4},
$$

where the units $\hbar=1$ and $m=1$ are used. Bound states of the system, that exist if $\lambda \geqslant 0$, correspond to those solutions of the Schrödinger equation

$$
\widehat{H} \phi(x)=E \phi(x)
$$

which vanish at infinity. Mc Weeny and Coulson noted [10] that the HO wavefunctions (4) with any value $\Omega>0$ constitute an appropriate basis in the RR calculations for the quartic AO, as they fulfil the bound-state condition, but the convergence of the scheme depends strongly on the value of $\Omega$. They observed that the approximation series for the $n$th state energy converges quickly if the value of $\Omega$ is fixed so as to minimize the matrix element $\langle n|H| n\rangle$. However, such a prescription works well only in the case of a single-well AO $\left(\omega^{2}>0\right)$ but fails if the potential is double-well shaped $\left(\omega^{2}<0\right)$ [11]. Moreover, diagonalization procedure has to be repeated with a different value of $\Omega$ for each considered state. A more effective strategy 
Table 1. The optimum value of the nonlinear parameter $\Omega_{\mathrm{opt}}^{(N)}$ determined from the trace condition and the corresponding relative error of the ground-state energy, $\delta E_{0}^{(N) \text { opt }}\left(\Omega_{\mathrm{opt}}\right)$, compared with $\Omega_{\text {min }}^{(N)}$, taken from [19], for which the relative error of ground-state energy is minimal, $\delta E_{0}^{(N)}\left(\Omega_{\min }\right)$.

\begin{tabular}{rllll}
\hline$N$ & $\sqrt{\Omega_{\mathrm{opt}}^{(N)}}$ & $\delta E_{0}^{(N)}\left(\Omega_{\mathrm{opt}}\right)$ & $\sqrt{\Omega_{\mathrm{min}}^{(N)}}$ & $\delta E_{0}^{(N)}\left(\Omega_{\min }\right)$ \\
\hline 1 & 1.29294233500847 & $1.09716442402927 \times 10^{-2}$ & 1.29294233500847 & $1.09716442402927 \times 10^{-2}$ \\
5 & 1.65920419620602 & $8.63597923540916 \times 10^{-7}$ & 1.70670645005687 & $1.33517756262170 \times 10^{-7}$ \\
10 & 1.86080663733626 & $1.86447406929386 \times 10^{-12}$ & 1.95004181438340 & $1.69012176051751 \times 10^{-13}$ \\
15 & 1.98940338014799 & $6.71884044353837 \times 10^{-19}$ & 2.09841072018140 & $2.7410652496 \times 10^{-19}$ \\
20 & 2.08593508969090 & $4.36194667514212 \times 10^{-24}$ & 2.20749144840388 & $4.9101 \times 10^{-25}$ \\
\hline
\end{tabular}

is to take approximations to all the desired states from diagonalization of a single RR matrix with a compromise value of $\Omega$. Many ways of fixing the value of $\Omega$ have been tested: so as to minimize the expectation value of the Hamiltonian in a chosen state of the HO basis (the first, namely $|0\rangle$ in [12], the central in [13], the last, namely $|N\rangle$ in [14], that one for which the expectation of the Hamiltonian is the smallest in [11]), so as to minimize a sum of expectation values in several HO states [15], or so as to vanish the matrix element $\langle 2|H| 0\rangle$ [16]. However, none of these prescriptions may be justified by the PMS, as none of the considered quantities does represent an approximation to a physical quantity. In this connection, one of us proposed [9] to fix the nonlinear parameters so as to make the trace of the truncated matrix stationary, since $\operatorname{Tr}_{N} H$ represents the $N$ th order approximation to the sum of energies of the $N$ lowest bound states. For the HO basis (4), this amounts to fixing the frequency in the $N$ th order calculation to the value $\Omega_{\mathrm{opt}}^{(N)}$, which fulfils

$$
\frac{\mathrm{d}}{\mathrm{d} \Omega} \operatorname{Tr}_{N} H=0 .
$$

As shown in [9], the scheme automatically yields well-converging results for the spectrum of the quartic AO, in both the single- and double-well cases. We may add here that the values $\Omega_{\mathrm{opt}}^{(N)}$, determined by the solution of (7), appear close to those for which the convergence of the above RR scheme is the quickest. This is demonstrated in table 1 , where $\Omega_{\mathrm{opt}}^{(N)}$ are compared with the values $\Omega_{\min }^{(N)}$, obtained by a numerical minimization of the error for the ground-state energy. A choice of nonlinear parameters has been considered on the same example of a quartic oscillator from a different perspective in [19], where an analytic formula for an optimum value has been derived from the asymptotic expansion. Comparing table 8 of [19] (where $\alpha$ corresponds to our $\Omega^{2}$ ) with our table 1 , we may see that the values derived from the asymptotic optimization scheme appear closer to $\Omega_{\text {min }}^{(N)}$ than our values $\Omega_{\text {opt }}^{(N)}$, but an extension of this scheme to other systems and other basis sets is problematic, since asymptotic expansions which are uniformly valid in the nonlinear parameters are difficult to construct. Whereas, a fully algorithmic formulation of our optimization scheme allows an easy application for arbitrary systems. Generally, the accuracy of the optimized RR method diminishes with increasing number of the level, but the energies of $N / 2$ lowest states can be trusted on, and using their values for calculating the free energy of the system, provides highly accurate results in a broad temperature range [9]. Similarly, the well-determined part of the spectrum has been successfully utilized for approximate description of the time evolution in the quartic oscillator potential [20].

It is worthwhile noting that the optimized RR scheme may be implemented in a way allowing an arbitrary precision calculation by taking advantage of the present computer algebra 
abilities to deal with exact numbers. As an example we use the Mathematica package to calculate energy difference $\Delta E$ between the first excited and the ground state of the doublewell oscillator with a Hamiltonian $\widehat{H}=-\frac{g}{2} \frac{\partial^{2}}{\partial x^{2}}+\frac{1}{2 g}\left(x^{2}-\frac{1}{4}\right)^{2}$ for $g=0.001$. Diagonalization of the RR matrix of order $N=350$ with the Mathematica precision constant set to 250 , yields the value of the level splitting

$$
\begin{aligned}
\Delta E= & 1.470464454175092501381989964494151981567800350052603 \\
& 52838605333378036605041575193505284182673433993282124674887 \\
& 3125608039469420665002677938176074660629119 \times 10^{-68},
\end{aligned}
$$

which agrees with the 225 digits quoted with the result obtained on the basis of Zinn-Justin conjecture and confirmed by high-precision power series method [21].

\subsection{Sextic oscillator bound states}

The optimized RR scheme performs also well for oscillators with higher than quartic anharmonicities, which present a more stringent test for approximation methods. We consider the sextic $\mathrm{AO}$ with a Hamiltonian given by

$$
\widehat{H}=-\frac{1}{2} \frac{\mathrm{d}^{2}}{\mathrm{~d} x^{2}}+\frac{\omega^{2}}{2} x^{2}+\lambda x^{6}
$$

that provides an interesting quasi-solvable example [22]. This is one of the rare cases when several bound states of a system may be obtained exactly, namely $p+1$ eigenstates are known if the parameters of the sextic oscillator satisfy the condition

$$
\omega^{2}=-(3+4 p+2 \nu) \sqrt{2 \lambda},
$$

where $v=0$ corresponds to the even-parity eigenstates and $v=1$ to the odd-parity ones. The detailed explanation of how to obtain the exact eigenvalues and the explicit form of the corresponding wavefunctions for a chosen value of $p$ is given in the appendix.

Bound-state energies $E(\omega, \lambda)$ depend on the parameters of the potential (9), however in calculating the numerical results, we may set $\lambda=1$ without loss of generality. The eigenenergies at other values of $\lambda$ can be obtained as multiples of $E\left(\omega \lambda^{-\frac{1}{4}}, 1\right)$, according to the scaling relation $E(\omega, \lambda)=\lambda^{\frac{1}{4}} E\left(\omega \lambda^{-\frac{1}{4}}, 1\right)$, obtained by applying the transformation $x \mapsto \lambda^{-\frac{1}{8}} x$ to the Schrödinger equation. As an example for testing the convergence of the optimized RR scheme we choose here the even-parity case with $p=8$, which according to (10) corresponds to the quasi-solvable Hamiltonian

$$
\left.\widehat{H}\right|_{\nu=0}=-\frac{1}{2} \frac{\mathrm{d}^{2}}{\mathrm{~d} x^{2}}-\frac{35 \sqrt{2}}{2} x^{2}+x^{6}
$$

with a family of nine exactly known states $(n=0,2, \ldots, 16)$. The $N$ th approximation to the AO bound states, $|n\rangle\rangle$, is obtained by numerical diagonalization of the Hamiltonian matrix in the basis of the $N$ lowest even-parity wavefunctions of the HO (4) with a frequency $\Omega_{\mathrm{opt}}^{(N)}$ determined from (7). In tables 2 and 3 the approximate values of energy and various moments of the position operator $x_{n}^{k(N)}\left(\Omega_{\mathrm{opt}}\right)=\left\langle\left\langle n\left|x^{k}\right| n\right\rangle\right\rangle$ are given for the ground state $(n=0)$ and for the highest of the analytically known excited states $(n=16)$, respectively. A quick convergence of all the moments in tables 2 and 3 indicates that wavefunctions are accurately determined. We have checked indeed that the agreement between the approximate and exact wavefunctions to graphical accuracy is obtained already at $N=15$. An exponential convergence for boundstate energies is evidenced in figure 1 , where the relative error $\delta E_{n}^{(N)}\left(\Omega_{\mathrm{opt}}\right)$ is plotted as a function of $N$. In the case of the Hamiltonian $\left.\widehat{H}\right|_{\nu=1}=-\frac{1}{2} \frac{\mathrm{d}^{2}}{\mathrm{~d} x^{2}}-\frac{37 \sqrt{2}}{2} x^{2}+x^{6}$, when the family 


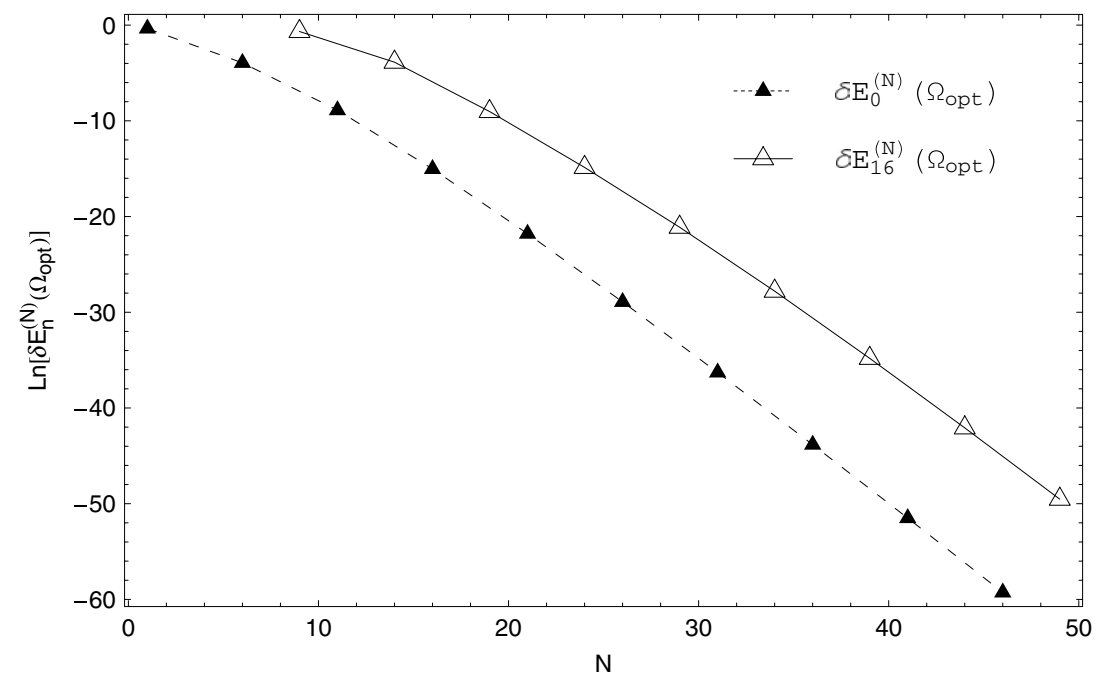

Figure 1. Semilogarithmic plot of the relative energy error, $\delta E_{n}^{(N)}\left(\Omega_{\mathrm{opt}}\right)$, for $n=0$ and $n=16$ states of the sextic oscillator (11) in function of the dimension $N$ of the optimized RR matrix. Here and in the following figures lines are drawn to guide the eye.

Table 2. The approximate energies $E_{0}^{(N)}\left(\Omega_{\mathrm{opt}}\right)$ and moments of the position operator $x_{0}^{k(N)}\left(\Omega_{\mathrm{opt}}\right)$ for $k=2,6,10$, obtained by means of the optimized RR method for the ground state $(n=0)$ of the sextic oscillator (11) at specific values of the dimension $N$. The underlined digits agree with the exact results.

\begin{tabular}{|c|c|c|c|c|}
\hline$N$ & $E_{0}^{(N)}\left(\Omega_{\mathrm{opt}}\right)$ & $x_{0}^{2(N)}\left(\Omega_{\mathrm{opt}}\right)$ & $x_{0}^{6(N)}\left(\Omega_{\mathrm{opt}}\right)$ & $x_{0}^{10(N)}\left(\Omega_{\text {opt }}\right)$ \\
\hline 20 & -40.52625280948697 & $\underline{2.72643166988877}$ & 23.60630747666730 & 242.23182746777999 \\
\hline 25 & -40.52625282342447 & 2.72643167388909 & 23.60630748254632 & 242.23182242437541 \\
\hline 30 & $-\underline{40.52625282343566}$ & $\underline{2.72643167389268}$ & 23.60630748253900 & $\underline{242.23182241788116}$ \\
\hline 35 & -40.52625282343567 & $\underline{2.72643167389269}$ & 23.60630748253899 & 242.23182241787521 \\
\hline
\end{tabular}

Table 3. Same as table 2, but for state $n=16$.

\begin{tabular}{lllll}
\hline$N$ & $E_{16}^{(N)}\left(\Omega_{\mathrm{opt}}\right)$ & $x_{16}^{2(N)}\left(\Omega_{\mathrm{opt}}\right)$ & $x_{16}^{6(N)}\left(\Omega_{\mathrm{opt}}\right)$ & $x_{16}^{10(N)}\left(\Omega_{\mathrm{opt}}\right)$ \\
\hline 20 & $\underline{40.52790329985854}$ & $\underline{2.03702842810644}$ & $\underline{35.34043326592744}$ & $\underline{856.72311216403564}$ \\
25 & $\underline{40.52625631425536}$ & $\underline{2.03737443716647}$ & $\underline{35.34277775827479}$ & $\underline{\underline{856.421838634955585}}$ \\
30 & $\underline{40.52625282743947}$ & $\underline{2.03737569324205}$ & $\underline{35.34280112924913}$ & $\underline{\underline{856.42125273736842}}$ \\
35 & $\underline{40.52625282343881}$ & $\underline{2.03737569518445}$ & $\underline{35.34280117889126}$ & $\underline{\underline{856.42125207650909}}$ \\
40 & $\underline{40.52625282343567}$ & $\underline{\underline{2.03737569518631}}$ & $\underline{35.34280117894960}$ & $\underline{856.42125207596973}$ \\
\hline
\end{tabular}

of nine odd-parity states is exactly known, the convergence properties of the optimized RR method are similarly good, but they slowly worsen with increasing value of $\omega$, when the double-well potential becomes deeper and more bound states are known exactly.

Also in the case of higher anharmonicities, the RR method optimized by the trace condition enables highly accurate determination of the spectrum in relatively low order calculation. For instance, diagonalization of matrices of order $N<100$ is sufficient for reobtaining all the bound states of the most difficult multi-well AO examples collected in [23] with the precision gradually diminishing from 50 digits for the ground state to 40 digits for the tenth excited 
state. The accuracy of various moments of the position operator is also good, as the hypervirial relations are fulfilled to a very high precision. Instead of presenting extensive tables of results, we refer the reader to our web page with the Mathematica program [24] which may be easily adapted for solving the desired AO problem to the desired precision.

\section{Spherically symmetric case}

The optimized RR method can be easily extended to the case of a central potential $V(r)$, where $r=|\vec{r}|$, when the Schrödinger equation reduces to the one-variable problem at a fixed angular momentum $l$. In the three-dimensional space, the problem is represented by the equation

$$
\left[-\frac{1}{2 r} \frac{\mathrm{d}^{2}}{\mathrm{~d} r^{2}} r+\frac{l(l+1)}{2 r^{2}}+V(r)\right] R(r)=E R(r),
$$

which upon introducing $u(r)=r R(r)$ is transformed to the form

$$
\widehat{H}^{(l)} u(r)=E u(r)
$$

with the radial Hamiltonian

$$
\widehat{H}^{(l)}=-\frac{1}{2} \frac{\mathrm{d}^{2}}{\mathrm{~d} r^{2}}+\frac{l(l+1)}{2 r^{2}}+V(r),
$$

and boundary conditions $u(r) \rightarrow 0$, as $r \rightarrow \infty$ and $u(0)=0$.

\subsection{Pseudoharmonic oscillator basis}

The basis for the RR method that is appropriate for spherically symmetric problems may be constructed from the set of eigenfunctions of the Hamiltonian

$$
\widehat{H}_{\mathrm{PHO}}=-\frac{1}{2} \frac{\mathrm{d}^{2}}{\mathrm{~d} r^{2}}+\frac{\Omega^{2} r^{2}}{2}+\frac{A}{2 r^{2}}
$$

which is exactly solvable, as shown by Davidson in 1930s [25] (pseudo-harmonic oscillator (PHO) or Goldman and Krivchenkov potential). The PHO has an equidistant energy spectrum

$$
E_{n}=\Omega(2 n+\gamma),
$$

where $\gamma=1+\frac{1}{2} \sqrt{1+4 A}$, and the normalized radial eigenfunctions of the form

$$
u_{n}^{\gamma, \Omega}(r)=(-1)^{n} \frac{1}{\Gamma(\gamma)} \sqrt{\frac{2 \Omega^{\gamma} \Gamma(\gamma+n)}{n !}} r^{\gamma-\frac{1}{2}} \mathrm{e}^{-\frac{1}{2} r^{2} \Omega}{ }_{1} F_{1}\left(-n ; \gamma ; \Omega r^{2}\right) .
$$

The above set of functions constitutes an orthonormal basis in the Hilbert space $L_{2}(0, \infty)$ of all square integrable functions over the interval $(0, \infty)$. The completeness of the set has been shown by Hall et al [26]. For $A=l(l+1)$, i.e. $\gamma=l+\frac{3}{2}$, the solution (17) reduces to that of a spherically symmetric $\mathrm{HO}$ with a Hamiltonian

$$
\widehat{H}_{\mathrm{HO}}=-\frac{1}{2} \frac{\mathrm{d}^{2}}{\mathrm{~d} r^{2}}+\frac{\omega^{2} r^{2}}{2}+\frac{l(l+1)}{2 r^{2}} .
$$

Optimization of nonlinear parameters of the PHO basis through the trace condition (3) amounts to choosing the values of $\Omega$ and $\gamma$ in the $N$ th order RR calculation so as to satisfy

$$
\frac{\mathrm{d}}{\mathrm{d} \Omega} \operatorname{Tr}_{N} H^{(l)}=0, \quad \text { and } \quad \frac{\mathrm{d}}{\mathrm{d} \gamma} \operatorname{Tr}_{N} H^{(l)}=0 .
$$




\subsection{Radial oscillators}

Among interesting examples that can be easily treated by the optimized RR method are the radial oscillators described by the Hamiltonian of the form

$$
\widehat{H}^{(l)}=-\frac{1}{2 r} \frac{\mathrm{d}^{2}}{\mathrm{~d} r^{2}} r+\frac{l(l+1)}{2 r^{2}}+\frac{\omega^{2} r^{2}}{2}+\lambda r^{k}
$$

with powers of anharmonicity $k$ being both positive and negative. The above Hermitian symmetric Hamiltonian is semi-bounded and consequently has self-adjoint extension in the Hilbert space $L_{2}(0, \infty)$ which admits a spectral decomposition [27]. Its spectrum is purely discrete. The Schrödinger equation for the above Hamiltonian may be rescaled in two ways: the transformation $r \mapsto r \lambda^{\frac{1}{(k+2)}}$ leads to the relation $E(\omega, \lambda)=\lambda^{\frac{2}{(k+2)}} E(z, 1)$ and the transformation $r \mapsto r \omega^{-\frac{1}{2}}$ yields $E(\omega, \lambda)=\omega E\left(1, z^{\frac{-(k+2)}{2}}\right)$ with the dimensionless parameter $z=\omega \lambda^{-\frac{2}{(k+2)}}$. This shows that power $k=-2$ sets the border between very different behavior of the oscillators. Denoting by $\kappa$ a positive value $\left|\frac{2}{(k+2)}\right|$, in the case of $k>-2$ we have $z=\omega \lambda^{-\kappa}$ and $E(\omega, \lambda)=\lambda^{\kappa} E(z, 1)$ and $E(\omega, \lambda)=\omega E\left(1, z^{-\kappa}\right)$, which shows that for $\lambda \rightarrow 0$ or $\omega \rightarrow 0$, the weak coupling or strong coupling perturbation theory may be respectively applied. The cases of $k<-2$ (spiked oscillators) are very different. For spiked oscillators, $z=\omega \lambda^{\kappa}, E(\omega, \lambda)=\lambda^{\kappa} E(z, 1)$ and $E(\omega, \lambda)=\omega E\left(1, z^{\kappa}\right)$, which indicate that neither of the two terms of the interaction potential may be taken as dominant and the conventional perturbation expansions cannot be applied. The Klauder phenomenon [28] does not occur for radial problems with the boundary conditions of the Dirichlet type, i.e. $u=0$ at the singular point $r=0$, and for $\lambda \rightarrow 0$ the energy eigenvalues of a spiked oscillator converge to those of the spherically symmetric HO of frequency $\omega(18)$. However, the perturbation series contains the terms logarithmic in $\lambda$ and is ordered in fractional powers of $\lambda$, thus unconventional methods have to be invoked for its derivation $[28,29]$. The perturbation theory with respect to $\widehat{H}_{\mathrm{HO}}$ is supersingular because the matrix elements $\left\langle m\left|r^{k}\right| n\right\rangle$ in the HO basis are infinite. Similar difficulties would be encountered for spiked oscillators in conventional RR calculations with the $\mathrm{HO}$ basis. As pointed out by Hall et al [30], the problem may be avoided by using the PHO basis with $\gamma>-k / 2$, since in this case the matrix elements appear finite and the RR calculations are well defined. For the anharmonic oscillators (20) the numerical calculations may be simplified by removing the dependence on $\Omega$ from the matrix elements by rescaling $r \mapsto \frac{r}{\sqrt{\Omega}}$, which yields

$$
\begin{aligned}
H_{m n}^{(l)}=(2 n+\gamma) & \Omega \delta_{m n}+\frac{\left(\omega^{2}-\Omega^{2}\right)}{2 \Omega}\left\langle m\left|r^{2}\right| n\right\rangle \\
& -\frac{\Omega}{2}\left(\gamma^{2}-2 \gamma+\frac{3}{4}-l(l+1)\right)\left\langle m\left|\frac{1}{r^{2}}\right| n\right\rangle+\frac{\lambda}{\Omega^{k / 2}}\left\langle m\left|r^{k}\right| n\right\rangle,
\end{aligned}
$$

where the ket $|n\rangle$ corresponds to the radial function (17) with $\Omega=1$, namely

$$
u_{n}^{\gamma}(r)=(-1)^{n} \frac{1}{\Gamma(\gamma)} \sqrt{\frac{2 \Gamma(\gamma+n)}{n !}} r^{\gamma-\frac{1}{2}} \mathrm{e}^{-\frac{1}{2} r^{2}}{ }_{1} F_{1}\left(-n ; \gamma ; r^{2}\right) .
$$

In this work we consider three different examples: the spherically symmetric sextic oscillator $(k=6)$, the harmonium potential $(k=-1)$ and the 'antisextic' spiked oscillator $(k=-6)$, which all enjoy the nice feature of quasi-exact solvability. We use the explicitly known solutions for testing the performance of the optimized RR method for both regular and singular spherically symmetric oscillators. In the case of positive power anharmonicities $(k>0)$, the optimum values of $\gamma$ turn out to be around $l+3 / 2$, we may put therefore $\gamma=$ $l+3 / 2$ and use the basis of the radial HO eigenfunctions with frequency $\Omega$ being the 
Table 4. The approximate energy $E_{0}^{(N)}\left(\Omega_{\mathrm{opt}}\right)$ and the moments of the radius operator $r_{0}^{k(N)}\left(\Omega_{\mathrm{opt}}\right)$ $(k=2,6,10)$ determined by the optimized RR method for the ground state $(n=0)$ of the radial sextic oscillator (25) for $l=1$.

\begin{tabular}{|c|c|c|c|c|}
\hline$N$ & $E_{0}^{(N)}\left(\Omega_{\mathrm{opt}}\right)$ & $r_{0}^{2(N)}\left(\Omega_{\mathrm{opt}}\right)$ & $r_{0}^{6(N)}\left(\Omega_{\mathrm{opt}}\right)$ & $r_{0}^{10(N)}\left(\Omega_{\mathrm{opt}}\right)$ \\
\hline 20 & $-\underline{48.11353417791531}$ & $\underline{2.90220193255671}$ & 27.98886643153595 & 314.77282893841708 \\
\hline 25 & -48.11353418904174 & 2.90220193690299 & 27.98886651687285 & 314.77282672623041 \\
\hline 30 & -48.11353418905196 & 2.90220193690737 & 27.98886651695577 & 314.77282672244758 \\
\hline 35 & -48.11353418905196 & 2.90220193690738 & 27.98886651695584 & 314.77282672244337 \\
\hline
\end{tabular}

Table 5. Same as table 4 , but for the state $n=8$.

\begin{tabular}{|c|c|c|c|c|}
\hline$N$ & $E_{8}^{(N)}\left(\Omega_{\mathrm{opt}}\right)$ & $r_{8}^{2(N)}\left(\Omega_{\mathrm{opt}}\right)$ & $r_{8}^{6(N)}\left(\Omega_{\mathrm{opt}}\right)$ & $r_{8}^{10(N)}\left(\Omega_{\mathrm{opt}}\right)$ \\
\hline 20 & 48.11737731862358 & 2.17521762647996 & 42.02668954998873 & 1136.40959750355092 \\
\hline 25 & 48.11354441726266 & 2.17592836963907 & 42.03132546920644 & 1135.67058214554528 \\
\hline 30 & 48.11353420292910 & 2.17593183660339 & 42.03139851074727 & 1135.66913552605609 \\
\hline 35 & 48.11353418906437 & $\underline{2.17593184311651}$ & 42.03139869962153 & $\underline{1135.66913419959634}$ \\
\hline 40 & 48.11353418905197 & $\underline{2.17593184312375}$ & 42.03139869987743 & 1135.66913419894809 \\
\hline
\end{tabular}

only parameter to be optimized. On the other hand, in the case of singular anharmonic oscillators $(k<-1)$, the role of the parameter $\gamma$ is crucial, and we show that the values of $\gamma_{\text {opt }}$ determined from the trace optimization are always greater than $-k / 2$, which ensures a successful calculation.

3.2.1. Radial sextic oscillator. First, we consider a sextic AO with the radial Hamiltonian of the form

$$
\widehat{H}^{(l)}=-\frac{1}{2} \frac{\mathrm{d}^{2}}{\mathrm{~d} r^{2}}+\frac{l(l+1)}{2 r^{2}}+\frac{\omega^{2}}{2} r^{2}+\lambda r^{6} .
$$

As shown in the appendix, the wavefunctions of the $p+1$ lowest states are known in a closed form if the parameters of the above oscillator are related as

$$
\omega^{2}=-(5+4 p+2 l) \sqrt{2 \lambda}
$$

which is similar to that for the odd-parity one-dimensional case (10) but includes in addition the orbital number $l$. Besides a normalization factor $\frac{1}{\sqrt{2}}$, the s-wave sector in a central potential is equivalent to the odd sector in a one-dimensional potential, therefore we need only consider the case $l>0$. Setting $\lambda=1$, we discuss the case of $p=8$, when

$$
\widehat{H}^{(l)}=-\frac{1}{2} \frac{\mathrm{d}^{2}}{\mathrm{~d} r^{2}}+\frac{l(l+1)}{2 r^{2}}+\frac{(37+2 l) \sqrt{2}}{2} r^{2}+r^{6},
$$

comparing the nine analytically determined eigenstates with the approximations obtained from diagonalization of the RR matrix in the basis of the radial $\mathrm{HO}$ eigenfunction with optimized frequency $\Omega$. The tables 4 and 5 contain our results for orbital number $l=1$ for the lowest $(n=0)$ and highest $(n=8)$ of exactly known states. It can be observed how the approximations to bound-state energies, $E_{n 1}^{(N)}\left(\Omega_{\mathrm{opt}}\right)$, and the moments $r_{n 1}^{k(N)}\left(\Omega_{\mathrm{opt}}\right)=\left\langle\left\langle n\left|r^{k}\right| n\right\rangle\right\rangle$ for $k=2,6,10$, approach the exact values with increasing $N$. In figure 2 the dependence of the relative energy error on $N$ is shown for both states. 


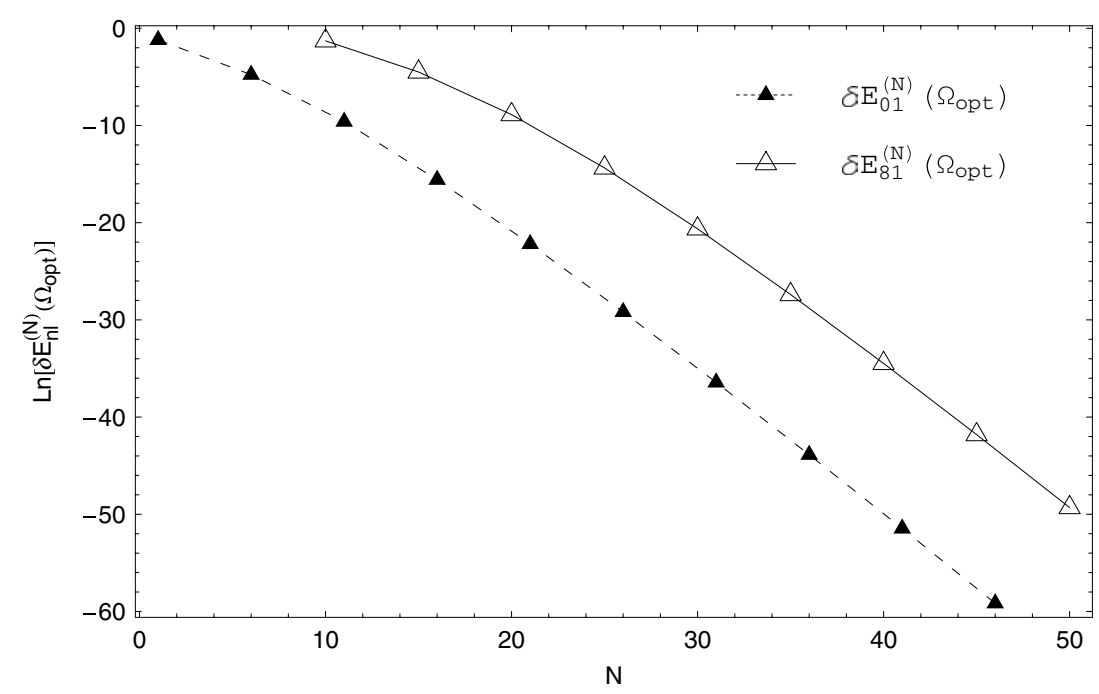

Figure 2. Semilogarithmic plot of the relative energy error, $\delta E_{n 1}^{(N)}\left(\Omega_{\mathrm{opt}}\right)$ for the two states $(n=0,8)$ of the radial sextic oscillator (25) as a function of the dimension $N$ of the optimized RR matrix.

3.2.2. Harmonium. The next example is related to the harmonium problem. Harmonium is a system of two particles confined in a harmonic potential and interacting via a Coulomb force, which enjoys the pleasant feature that the center of mass and the relative motion can be separated. The center of motion is subjected to a solvable harmonic oscillator equation, and the spherically symmetric relative motion equation corresponds to the $k=-1$ power AO with a radial Hamiltonian of the form

$$
\widehat{H}^{(l)}=-\frac{\mathrm{d}^{2}}{\mathrm{~d} r^{2}}+\frac{l(l+1)}{r^{2}}+\omega^{2} r^{2}+\frac{\lambda}{r} .
$$

The observation that the above Hamiltonian possesses a closed-form solution [31, 32] was of great importance and thus provides further rationale for investigation of approximation methods in the many-body theory. The exact solution exists if for some integer $p$, the frequency $\omega$ fulfils the condition

$$
E=(3+2 l+2 p) \omega,
$$

which, in contrast to (24), does not depend on the coupling $\lambda$ but on the energy of the investigated state. In difference with the case of the sextic oscillator, for harmonium there is but a single bound state, not necessarily the ground state, that is known exactly. This happens if the parameters $\omega$ and $\lambda$ are related by a particular relation that depends on an integer $p$, as given in appendix (A.5). By utilizing the scaling property $E(\omega, \lambda)=\lambda^{2} E\left(\omega \lambda^{-2}, 1\right)$ and setting $\lambda=1$ we have that equation (A.5) determines the values $\omega_{p}$ at which the bound state is analytically known. Using the exact bound-state solutions for testing the convergence of the optimized RR method in the PHO basis, we compare three ways of fixing the nonlinear parameters: first, a naive RR scheme $\left(\Omega=\omega_{p}\right.$ and $\left.\gamma=3 / 2+l\right)$, second, optimization of the parameter $\Omega\left(\Omega=\Omega_{\text {opt }}\right.$ and $\left.\gamma=3 / 2+l\right)$ and third, optimization of both parameters ( $\Omega=\Omega_{\text {opt }}$ and $\gamma=\gamma_{\text {opt }}$ ). Figure 3 presents the semilogarithmic plot of the relative energy error $\delta E_{n l}^{(N)}$ as a function of $N$ for the largest possible value of confining frequency for which the ground-state solution is known, namely $\omega_{1}=0.25(l=0, p=1)$. A similar plot for a 


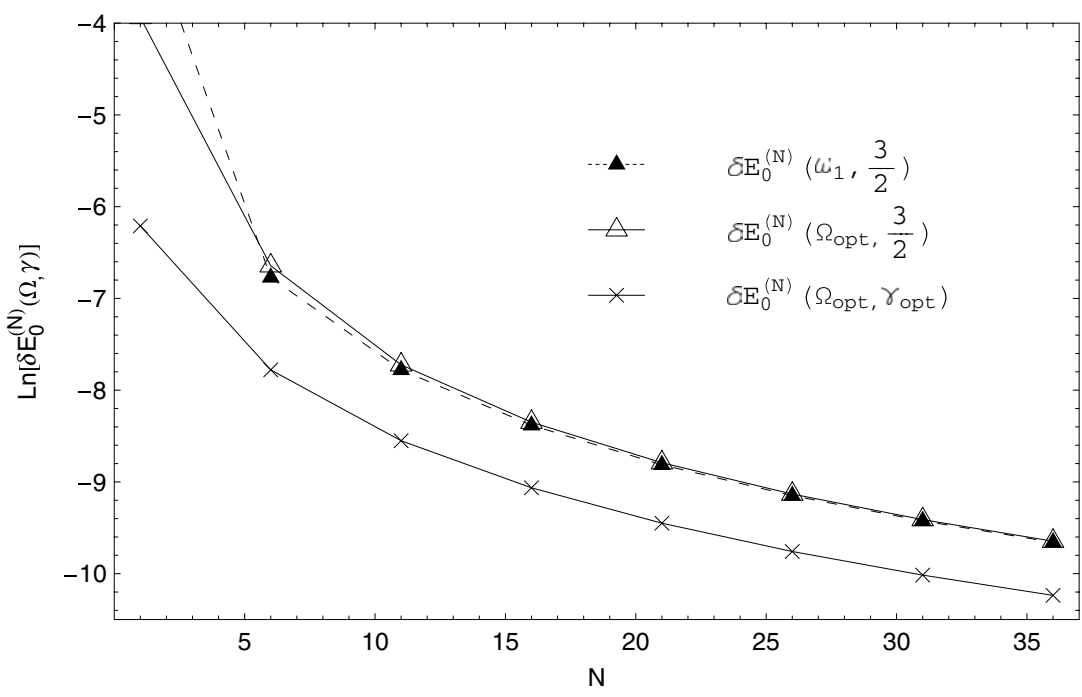

Figure 3. Semilogarithmic plot of the relative energy error for the ground state of harmonium with the confinement frequency $\omega_{1}=0.25$ as a function of the dimension $N$ of the optimized RR matrix.

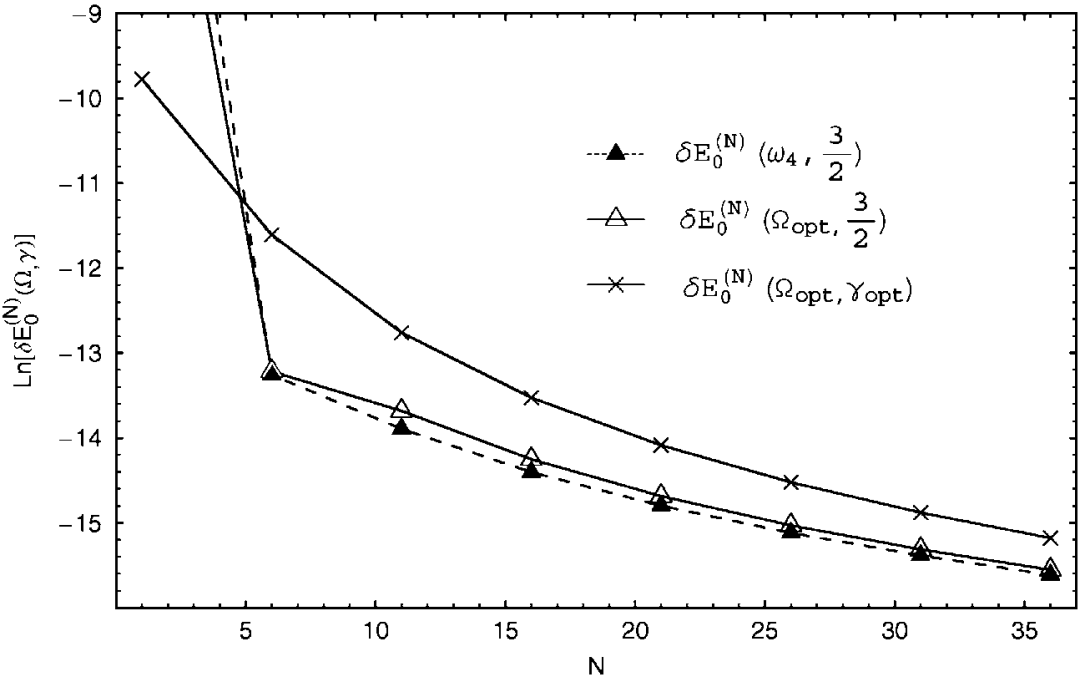

Figure 4. Like figure 3 , but for confinement frequency $\omega_{4} \approx 0.00867$.

smaller value of frequency, $\omega_{4}=\frac{35-3 \sqrt{57}}{1424} \approx 0.00867(l=0, p=4)$, is given in figure 4 . The results for higher values of angular momentum $l$ show a similar tendency, therefore we do not show them. In the case of large confinement frequency the optimized scheme proves superior over the naive one, but in the strong correlation limit $(\omega \ll 1)$ it is the naive one that works slightly better. We may notice that the application of the trace condition in the RR scheme ensures an exponential convergence, albeit its rate is generally slower than in the case 
of positive power AOs. Since the values of nonlinear parameters determined from the trace condition appear close to $\gamma=3 / 2+l$ and $\Omega=\omega_{p}$, the optimized RR scheme may be regarded as a justification for using the naive RR method for harmonium-like systems.

3.2.3. Spiked oscillator. Now, we come to the computationally more difficult case of a spiked oscillator with anharmonic potential of the power $k<-2$, which exhibits a highly singular behavior at the origin. As an example we consider the 'antisextic' oscillator $(k=-6)$ with the radial Hamiltonian given by

$$
\widehat{H}^{(l)}=-\frac{1}{2} \frac{\mathrm{d}^{2}}{\mathrm{~d} r^{2}}+\frac{l(l+1)}{2 r^{2}}+\frac{\omega^{2}}{2} r^{2}+\frac{\lambda}{r^{6}} .
$$

A bound state of the system may be considered analytically determined if its energy is given by

$$
E^{(p)}=2(p+1) \omega
$$

and a specific relation between $\omega$ and $\lambda$ (A.16) is satisfied for an integer $p$. As opposed to the previously discussed sextic oscillator and harmonium cases, where the results have been rescaled in terms of $\lambda$, in the case of spiked oscillators we exploit the rescaling in terms of $\omega$, namely $E(\omega, \lambda)=\omega E\left(1, \omega^{2} \lambda\right)$. This allows us to put $\omega=1$ in the numerical calculation in accordance with the usual practice in the literature on spiked oscillators. The specific values of $\lambda$ at which the bound states are analytically known may be obtained by substituting (A.17) into (A.16) and determining the $p+1$ solutions of the polynomial equation (A.5). If $\lambda_{p(i)}$ are numbered with decreasing value of $\lambda$ from $i=0$ to $i=p$, then the $i$ th exactly known state represents the $i$ th excitation in the respective potential.

The PHO basis has been used in the RR calculations for spiked oscillators by Hall et al [26], who derived useful formulae for the matrix elements of the operator $r^{k}$ in the PHO basis on condition of $\gamma>-k / 2$. In the case of the 'antisextic' oscillator this amounts to $\gamma>3$, and the expressions for matrix elements $\left\langle m\left|\frac{1}{r^{6}}\right| n\right\rangle$ are singular at the values $\gamma=0,1,2,3$. Hall et al [26] used the prescription of fixing nonlinear parameters $\left(A=\gamma^{2}-2 \gamma+\frac{3}{4}\right.$ and $B=\Omega^{2}$ ) so as to minimize the approximate energy of the considered level $E_{n l}^{(N)}(\Omega, \gamma)$. In low orders, an algebraic diagonalization of the RR matrix has allowed them to obtain analytical approximations to ground-state energy of the 'antisextic' oscillator, but for its precise determination in higher order calculation, a time-consuming procedure of iterative optimization of nonlinear parameters must have been used by Saad et al [5]. Our scheme requires much less computational cost, since the optimum values of nonlinear parameters are determined from the trace condition and further diagonalization of the RR matrix is performed only once in each order calculation. The convergence of our method is demonstrated on two quasi-solvable examples of the 'antisextic' oscillator with angular momentum $l=0$, where ground-state energies are analytically known. The semilogarithmic plot of relative errors is shown in figure 5 for $p=2$ case $\left(\lambda_{2(0)}=\frac{5}{384}\left[9887+32 \sqrt{333778} \cos \left[\frac{1}{3} \arctan \left(\frac{1852389 \sqrt{1001}}{478512623}\right)\right]\right] \approx 369.26, E_{00}^{(2)}=6\right)$, and in figure 6 for $p=0$ case $\left(\lambda_{0(0)}=\frac{9}{128} \approx 0.07, E_{00}^{(0)}=2\right)$. We observe that for both large and small values of $\lambda$, the results of the two-parameter $\left(\Omega_{\mathrm{opt}}, \gamma_{\mathrm{opt}}\right)$ and the one-parameter optimization $\left(\Omega=\omega=1, \gamma_{\mathrm{opt}}\right)$ are nearly the same. We have checked that for other spiked oscillators the case is similar and the calculations may be simplified, since only the parameter $\gamma$ needs to be optimized. This is in difference with the results of the approach that utilizes iterative optimization of ground-state energy, presented in table 2 of [5], where much quicker convergence has been obtained by optimizing both parameters. However, plotting the relative errors for exited states determined from diagonalization of the RR matrix with the values of 


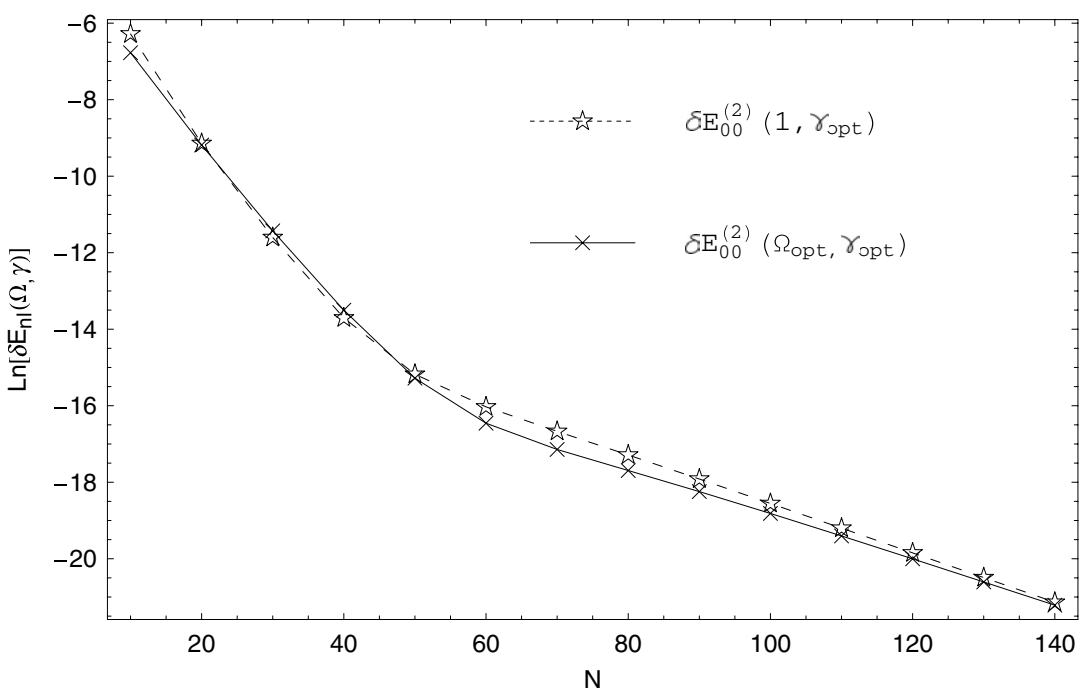

Figure 5. Semilogarithmic plot of the relative energy error for the ground state of the radial spiked oscillator $(28)$ of the strength $\lambda_{2(0)} \approx 369.26$ as a function of the dimension $N$ of the optimized RR matrix.

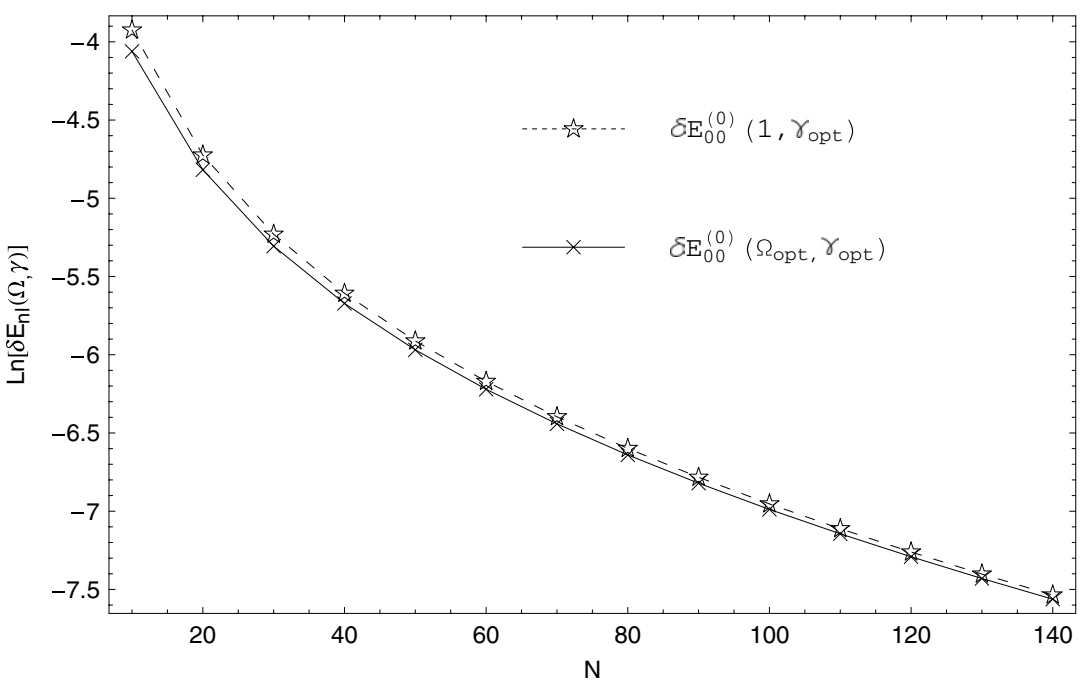

Figure 6. Like figure 5 , but for the strength $\lambda_{0(0)} \approx 0.07$.

parameters taken from table 2 of [5] for the case of $\lambda=0.1$ in figure 7 , we observe that the precision of energy determination in this approach rapidly decreases as the number of the level increases. In contrast, the results of our approach, obtained from the RR matrix of the same dimension $N=80$, plotted on the same figure, indicate a uniformly good precision in a wide range of energy levels. We conclude that the values of nonlinear parameters obtained from minimization of the trace of the RR matrix are appropriate for a precise determination of the whole part of the spectrum, although this is not necessarily the best possible choice for a particular level. 


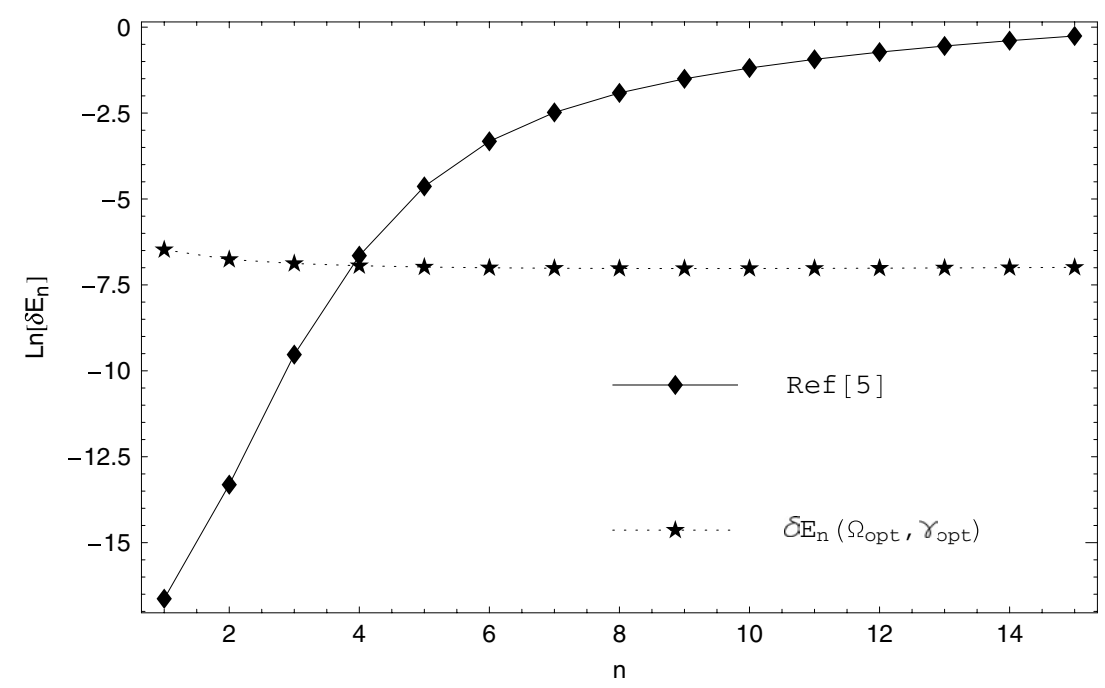

Figure 7. The semilogarithmic plot of the relative error of bound state energy of the spiked oscillator of $\lambda=0.1$ as a function of the level number $n$, determined by the method of [5] and by the optimized RR method of the present work.

Table 6. The approximate energy $E_{0}^{(N)}\left(\gamma_{\mathrm{opt}}\right)$ and the moments of the radius operator $r_{0}^{k(N)}\left(\gamma_{\mathrm{opt}}\right)$ $(k=1,2,6)$ determined in the optimized RR method for the ground state of the radial spiked oscillator (28) of the strength $\lambda_{2(0)} \approx 369.26$.

\begin{tabular}{|c|c|c|c|c|c|}
\hline$N$ & $\gamma_{\mathrm{opt}}$ & $E_{0}^{(N)}\left(\gamma_{\mathrm{opt}}\right)$ & $x_{0}^{(N)}\left(\gamma_{\mathrm{opt}}\right)$ & $x_{0}^{2(N)}\left(\gamma_{\mathrm{opt}}\right)$ & $x_{0}^{6(N)}\left(\gamma_{\mathrm{opt}}\right)$ \\
\hline 20 & 14.48 & 6.00021390368223 & 2.84582121384655 & 8.28291890988045 & 737.684487549365 \\
\hline 40 & 17.29 & $\overline{6.00000223509568}$ & $\overline{2.84562114647890}$ & $\overline{8.28} 186647605726$ & $\overline{737} .249285331635$ \\
\hline 80 & 20.97 & 6.00000006213529 & 2.84561903526830 & 8.28185556086959 & $\overline{737.240961117416}$ \\
\hline 120 & 23.61 & 6.00000000480818 & 2.84561898882020 & $\underline{8.28185531621157}$ & 737.240889729308 \\
\hline Exact & & $\overline{6}$ & 2.84561898466095 & 8.28185529459909 & $\overline{737.240860856683}$ \\
\hline
\end{tabular}

Table 7. Like table 6 , but for the strength $\lambda_{0(0)} \approx 0.07$.

\begin{tabular}{llllll}
\hline$N$ & $\gamma_{\mathrm{opt}}$ & $E_{0}^{(N)}\left(\gamma_{\mathrm{opt}}\right)$ & $r_{0}^{(N)}\left(\gamma_{\mathrm{opt}}\right)$ & $r_{0}^{2(N)}\left(\gamma_{\mathrm{opt}}\right)$ & $r_{0}^{6(N)}\left(\gamma_{\mathrm{opt}}\right)$ \\
\hline 20 & 4.38 & $\underline{2.01776622625948}$ & $\underline{1.44367633236718}$ & $\underline{2.28981024534251}$ & 30.6645428912254 \\
40 & 5.02 & $\underline{\underline{2.0}} 734163831413$ & $\underline{1.43695960594035}$ & $\underline{2.27129896763614}$ & $\underline{29.8195939603567}$ \\
80 & 5.88 & $\underline{\underline{2.00}} 272386999177$ & $\underline{\underline{1.43}} 401033592011$ & $\underline{2.26321009528890}$ & $\underline{\underline{29}} .6572447265661$ \\
120 & 6.50 & $\underline{\underline{2.00}} 140501517273$ & $\underline{\underline{1.43}} 316756712602$ & $\underline{2.26090435812050}$ & $\underline{29} .7830590372660$ \\
Exact & & 2 & 1.43226578557733 & 2.25844053161144 & 29.4482015786915 \\
\hline
\end{tabular}

In tables 6 and 7 we show the numerical results of the RR method with the parameter $\gamma$ optimized through minimization of the trace for the above-discussed quasi-solvable cases. One can observe how the values of ground-state energy and various moments of the radial position operator converge to the exact values with increasing dimension of the RR matrix $N$. The optimum value of $\gamma$ depends strongly on $\lambda$ and grows with $N$. In all the cases we studied, the condition $\gamma_{\mathrm{opt}}>3$ is fulfilled, although the smaller the $\lambda$ is, the closer the $\gamma_{\mathrm{opt}}$ is to the 

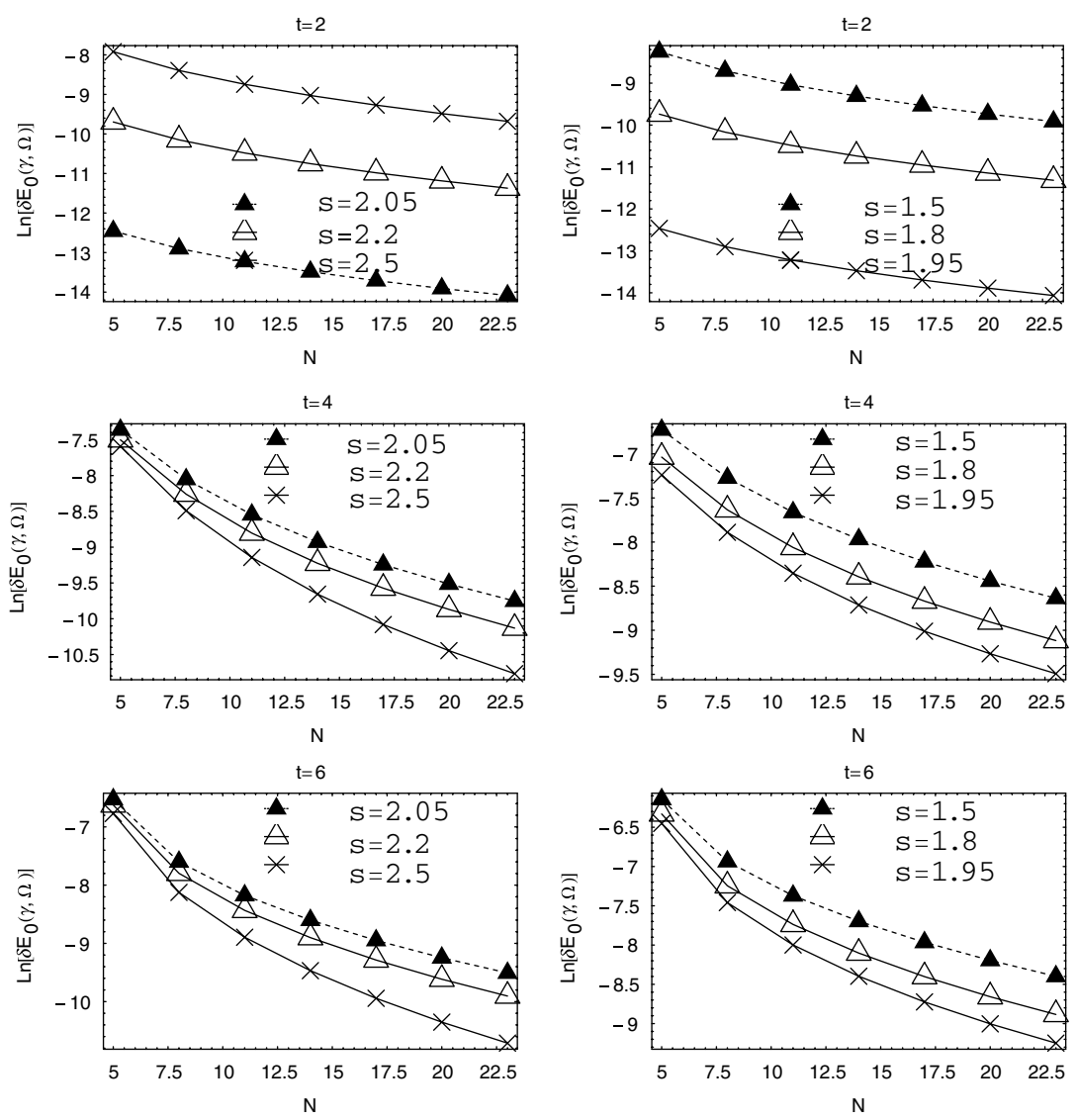

Figure 8. The semilogarithmic plot of the relative error of the optimized RR method for bound state energies of the generalized oscillator $V(r)=r^{s}+r^{t}$ for various powers $s$ and $t$, as a function of the dimension $n$.

value 3, where the matrix elements become singular, which explains the heavy worsening of convergence with decreasing $\lambda$. Nevertheless, we may observe that for $\lambda \rightarrow 0$ the bound-state energies approach smoothly those of the radial HO, which is due to using the PHO basis that ensures the satisfaction of the Dirichlet boundary condition in the RR calculation.

3.2.4. Generalized oscillators. We have tested the convergence properties of our approach further by considering the potential $V(r)$ to be a linear combination of $r^{s}$ and $r^{t}$ with the power $s$ being negative and t being positive. The optimized RR method does allow solution to eigenvalue problem for various combinations of potential parameters. In figure 8 we have plotted the error of ground-state energy determination for the exemplary potential $V(r)=r^{s}+r^{t}$ with various powers $s=-1.5,-1.8,-1.95,-2.05,-2.2,-2.5$ and $t=2,4,6$ in function of the dimension of the optimized RR matrix, $N$. The convergence becomes exponential at not too large values of $N$, with the rate depending on the detailed shape of the potential. In all the cases considered, using the PMS condition for the trace of the RR matrix for fixing the nonlinear parameters $\Omega$ and $\gamma$ ensures an effective determination of the spectrum. 


\section{Conclusion}

We have discussed optimization of the RR scheme by introducing nonlinear parameters, where values are fixed by minimization of the trace of the truncated matrix. Using the basis of the HO eigenfunctions with optimized frequency $\Omega$, we obtain an efficient method for determining spectrum of multi-well one-dimensional AOs to practically any precision. In the case of radial oscillators with $\lambda r^{k}$ anharmonicity, the basis of the PHO eigenfunctions with two arbitrary parameters $\Omega$ and $\gamma$ seems more suitable. For positive power oscillators $(k>0)$ the role of the parameter $\gamma$ turns out to be minor, and the scheme may be simplified by using the radial HO eigenfunctions (i.e., setting $\gamma=3 / 2+l$ ). In the case of negative power oscillators $k<-1$, the parameter $\Omega$ plays a minor role and may be set equal to the frequency $\omega$ of the harmonic term in the Hamiltonian, whereas optimization of the parameter $\gamma$ in each order calculation is crucial for a good convergence. In the limiting case of harmonium-like potential $(k=-1)$ the optimum values of both parameters turn out to be equal to those that are used in the naive RR method $(\Omega=\omega$ and $\gamma=3 / 2+l)$. The optimized RR method performs well not only for energies but also for wavefunctions, yielding well-convergent approximations to various moments of the position operator.

The RR method optimized by the trace condition appears effective for numerical calculation and may be used for arbitrary accuracy within the modern software environment. It turns out that far greater improvement of accuracy is obtained from optimizing the nonlinear parameters in each order calculation by the trace condition rather than from increasing the dimension of the basis with the parameters remaining fixed. The computational cost of our scheme is much lower than in the case of iterative optimization of nonlinear parameters. Another advantage is that the whole set of energy levels may be determined at once and the approximate eigenvectors are mutually orthogonal. For the class of potentials with the purely discrete spectrum considered in the present work, the RR method with the PHO basis is highly competitive with existing methods, the results of which are easily recovered in our approach. The convergence of our approach may be slower than that achieved in the methods specialized for a particular problem, but the advantage is that the results are obtained automatically for a large class of systems by the algorithm described above without the necessity of specifying any starting value.

\section{Appendix. Quasi-exact solutions}

In the appendix we consider the problem of quasi-solvability of anharmonic oscillators, thereby deriving the formulae for the analytically known solutions that are used for testing the convergence of the optimized RR method. Generally, the problem is quasi-solvable if the eigenfunction can be represented as

$$
\psi(y)=f(y) \sum_{n=0}^{p} a_{n} y^{n},
$$

and the coefficients of the series satisfy the three-term recurrence relation

$$
A_{n} a_{n+1}+B_{n} a_{n}+C_{n} a_{n-1}=0, \quad n=0,1,2, \ldots
$$

where $a_{-1}=0$. The coefficients of the series $a_{n}$ as well those of the recurrence $A_{n}, B_{n}, C_{n}$ depend solely on the parameters of the potential and the bound-state energy $E$. In order for the series in (A.1) to terminate after the $p$ th term, we must have

$$
C_{p+1}=0
$$


and

$$
a_{p+1}=0 .
$$

It is easy to convince oneself [33] that the second condition is equivalent to

$$
\operatorname{Det}\left(\begin{array}{ccccc}
B_{0} & A_{0} & \ldots & \ldots & 0 \\
C_{1} & B_{1} & A_{1} & \ldots & 0 \\
0 & C_{2} & B_{2} & A_{2} & 0 \\
\ldots & \ldots & \ldots & \ldots & \ldots \\
0 & \ldots & C_{p-1} & B_{p-1} & A_{p-1} \\
0 & \ldots & \ldots & C_{p} & B_{p}
\end{array}\right)=0,
$$

where the determinant is a polynomial of the degree $p+1$ in the variable $E$. The two equations (A.3) and (A.5) may be used to determine the specific relations between the parameters of the potential that must be satisfied for the exact solution to be of the form (A.1).

\section{A.1. One-dimensional sextic oscillator}

For the one-dimensional sextic $\mathrm{AO}(9)$ the analytically known eigenfunction, in the even-parity $(v=0)$ and odd-parity $(v=1)$ case, may be represented as

$$
\psi^{\nu}(x)=\mathrm{e}^{-\frac{\lambda x^{4}}{2 \sqrt{2}}} \sum_{n=0}^{p} a_{n} x^{2 n+\nu}
$$

where the coefficients of the series satisfy the recurrence relation (A.2) with

$$
\begin{aligned}
& A_{n}=(2 n+2+v)(2 n+1+v), \\
& B_{n}=2 E, \\
& C_{n}=-\omega^{2}-(4 n-1+2 v) \sqrt{2 \lambda} .
\end{aligned}
$$

The series in (A.6) terminates after the $p$ th term, if $C_{p+1}=0$ and $a_{p+1}=0$. The first condition is fulfilled if the parameters of the sextic oscillator satisfy

$$
\omega^{2}=-(3+4 p+2 v) \sqrt{2 \lambda} .
$$

For a chosen value of $p$, this corresponds to two quasi-solvable cases of a sextic oscillator: (i) $v=0$, where the $p+1$ lowest even-parity states are known; and (ii) $v=1$, where the $p+1$ lowest odd-parity states are known. In both cases, we can obtain the $p+1$ exact boundstate energies $E(\omega, \lambda)$ by solving the polynomial equation (A.5), and thus the corresponding exact wavefunctions by determining the non-vanishing coefficients $a_{n}$ from the recurrence relation (A.2).

\section{A.2. Radial sextic $A O$}

For the spherically symmetric sextic AO (23) the analytically known eigenfunction becomes

$$
u(r)=r^{l+1} \mathrm{e}^{-\frac{\lambda r^{4}}{2 \sqrt{2}}} \sum_{n=0}^{p} a_{n} r^{2 n},
$$

where the coefficients $a_{n}$ satisfy the relation (A.2) with recurrence coefficients

$$
\begin{aligned}
& A_{n}=2(n+1)(3+2 n+2 l), \\
& B_{n}=2 E, \\
& C_{n}=-\omega^{2}-(1+4 n+2 l) \sqrt{2 \lambda} .
\end{aligned}
$$


The closed-form solutions exist if the sextic oscillator parameters satisfy

$$
\omega^{2}=-(5+4 p+2 l) \sqrt{2 \lambda}=0
$$

and the $p+1$ bound-state energies are determined by the condition (A.5) with recurrence coefficients of the form (A.10).

\section{A.3. Harmonium}

The eigenfunction of the harmonium-like Hamiltonian (26) can be written as

$$
u(r)=r^{l+1} \mathrm{e}^{-\frac{\omega}{2} r^{2}} \sum_{n=0}^{p} a_{n} r^{n},
$$

where $a_{n}$ satisfy the relation (A.2) with recurrence coefficients

$$
\begin{aligned}
& A_{n}=(n+1)(n+2 l+2), \\
& B_{n}=-\lambda, \\
& C_{n}=E-(1+2 n+2 l) \omega .
\end{aligned}
$$

The closed-form solution is obtained if $C_{p+1}=0$, which means that

$$
E=(3+2 l+2 p) \omega
$$

and the condition (A.5) is satisfied with the recurrence coefficients given by (A.13). Compared to the anharmonic oscillator case, the condition (A.14) depends on energy; therefore for a particular value of $\omega$, denoted by $\omega_{p}$, only the bound state with energy $E_{p}=(1+2 n+2 l) \omega_{p}$ is known exactly.

\section{A.4. Spiked oscillator}

For the spiked AO with the radial Hamiltonian of the form (28), the analytically known eigenfunction assumes the form

$$
u(r)=r^{\frac{3}{2}} \mathrm{e}^{-\frac{\sqrt{2 \lambda}}{2 r^{2}}-\frac{\omega r^{2}}{2}} \sum_{n=0}^{p} a_{n} r^{2 n},
$$

where the coefficients $a_{n}$ satisfy the relation (A.2) with recurrence coefficients given by

$$
\begin{aligned}
& A_{n}=-4 \sqrt{2 \lambda}(n+1) \\
& B_{n}=-\frac{3}{4}-4 n(1+n)+l(l+1)+2 \sqrt{2 \lambda} \omega \\
& C_{n}=-2(E-2 n \omega) .
\end{aligned}
$$

Closed-form solutions are obtainable if

$$
E=2(p+1) \omega
$$

and the condition (A.5) is satisfied with recurrence coefficients as stated in (A.16). For a fixed $p$, equation (A.5) possess $p+1$ solutions that determine the $p+1$ cases of specific relations between $\lambda$ and $\omega$, at which the bound state of energy (A.17) is analytically known. 


\section{References}

[1] Reed M and Simmons B 1978 Methods of Modern Mathematical Physics vol 4 (New York: Academic) p 82

[2] Hylleraas E A 1929 Z. Phys. 54347

[3] Kołos W, Roothaan C C J and Sack R A 1960 Rev. Mod. Phys. 32178

[4] Cencek W, Komasa J and Rychlewski J 2005 Handbook on Parallel and Distributed Processing (Berlin: Springer) p 505

[5] Saad N, Hall R L and Katatbeh Q D 2005 J. Math. Phys. A 46 022104-1

[6] Stevenson P 1981 Phys. Rev. D 232961

[7] Cashwell W E 1979 Ann. Phys., NY 123153

[8] Killingbeck J P 1981 J. Phys. A: Math. Gen. 141005

[9] Okopińska A 1987 Phys. Rev. D 361273

[10] McWeeny R and Coulson C A 1948 Proc. Camb. Philos. Soc. 44413

11] Balsa R, Plo M, Esteve J G and Pacheco A F 1983 Phys. Rev. D 281945

[12] Jafarpour M and Afshar D 2002 J. Phys. A: Math. Gen. 3587

[13] Van der Straeten E and Nauds J 2006 J. Phys. A: Math. Gen. 39933

[14] Bishop R F, Flynn M F, Boscà M C and Guardiola R 1989 Phys. Rev. D 406154

[15] Quick R M and Miller H G 1985 Phys. Rev. D 312682

[16] Chaudhuri R N and Mondal M 1989 Phys. Rev. A 406080 Chaudhuri R N and Mondal M 1991 Phys. Rev. A 433241

[17] Killingbeck J P 2000 J. Phys. A: Math. Gen. 356999

[18] Korsch H J 2002 Eur. J. Phys. 23413

[19] Hill R N 1995 Phys. Rev. A 514433

[20] Amore P et al 2005 Phys. Lett. A 34087

[21] Alhendi H A and Lashin E I 2005 J. Phys. A: Math. Gen. 386785

[22] Chaudhuri R N and Mukherjee B 1984 J. Phys. A: Math. Gen. 173327

[23] Bandyopadhyay K, Bhattacharyya K and Bhattacharyya A K 2003 Phys. Lett. 31488

[24] http://www.pu.kielce.pl/strony/Anna.Okopinska/programs.html

[25] Davidson P M 1932 Proc. R. Soc. Lond. 130459

[26] Hall R L, Saad N and Keviczky A Bvon 2002 J. Math. Phys. A 4394

[27] Riesz F and Nagy B Sz 1965 Functional Analysis (New York: Ungar) section 124

[28] Detwiler L C and Klauder J R 1975 Phys. Rev. D 111436

[29] Harrell E M 1977 Ann. Phys., NY 105379

[30] Hall R L and Saad N 2000 J. Phys. A: Math. Gen. 33569

[31] Kais S, Herschbach D R and Levine R D 1989 J. Chem. Phys. 917791

[32] Taut M 1993 Phys. Rev. A 483561

[33] Bose S K and Gupta N 1998 Nuovo Cimento B 113299 